\title{
Sparse Representation Optimization of Gaussian Mixed Feature of Image Based on Convolution Neural Network
}

\author{
Yuguang Ye ( $\nabla$ chinaye153@163.com ) \\ Quanzhou Normal University
}

\section{Research Article}

Keywords: Convolutional neural network, image processing, high speed hybrid, data mining, sparse feature

Posted Date: October 18th, 2021

DOl: https://doi.org/10.21203/rs.3.rs-912092/v1

License: (c) (i) This work is licensed under a Creative Commons Attribution 4.0 International License. Read Full License

Version of Record: A version of this preprint was published at Soft Computing on January 6th, 2022. See the published version at https://doi.org/10.1007/s00500-021-06587-3. 


\title{
Sparse Representation Optimization of Gaussian Mixed Feature of Image Based on Convolution Neu- ral Network
}

\author{
Yuguang Ye $e^{1,2,3}$ \\ 1. Quanzhou Normal University, Quanzhou, Fujian,362000, China \\ 2. Fujian Provincial Key Laboratory of Data Intensive Computing, Quanzhou, Fujian,362000, China \\ 3. Key Laboratory of Intelligent Computing and Information Processing, Fujian Province University, Quanzhou, Fujian, 362000, \\ China
}

E-mail :chinaye153@163.com

\begin{abstract}
With the rapid development of intelligent algorithm and image processing technology, the limitations of traditional image processing methods are more and more obvious. Based on this, this paper studies a new pattern of sparse representation optimization of image Gaussian mixture feature based on convolution neural network, and designs a sparse representation system model of vehicle detection image based on convolution neural network. The vehicle image data is collected from many aspects, and the convolution neural network is used for comprehensive analysis and evaluation. The model can extract the feature information of the vehicle detection image better by making the scheme of the real-time vehicle detection image and according to the image features and convolution neural network algorithm. The results show that the Gaussian mixture feature sparse representation optimization model based on convolution neural network has the advantages of high feasibility, high data accuracy and high response speed, which can enhance the processing efficiency of vehicle detection image and improve the utilization of local environmental information in the image.
\end{abstract}

Keywords: Convolutional neural network, image processing, high speed hybrid, data mining, sparse feature

\section{Introduction}

At present, the traditional "many to many" cluster point information collection is the main way of vehicle image information in China [1]. Since the beginning of the 21st century, the rapid development of a variety of information technologies in China has also led to the transformation of obtaining source data from vehicle images. The emergence of modern vehicle navigation methods, such as intelligent path planning, efficient map navigation, and the combination of software and hardware, has provided opportunities for promoting "intelligent travel" on a large scale High efficiency has become an important feature of intelligent vehicle navigation in China [2]. At present, the existing image analysis system provides a large number of data information extraction schemes, but in the process of image processing, it is difficult to select a targeted sparse representation scheme according to its image local feature information to achieve the optimal analysis effect [3]. Xiang $\mathrm{T}$ and others found that the information extraction problem of vehicle detection image also takes the basic scheme formulation, traffic light network monitoring points and road condition features as the main dividing line, and the image sparse representation result is very poor to the traditional image decomposition quality [4]. Pan and other scholars have optimized the thinning process according to the image principle of threedimensional space, and proposed that we should pay attention to the development of three-dimensional basic structure or image decomposition algorithm based on Gaussian mixture, strengthen the development and construction of path planning and language tips based on three-dimensional space, and enhance the cognition and attention of intelligent navigation, The training of algorithm engineers should be improved [5]. According to the theory of multi factor relationship in collaboration, Miao Q and other scholars proposed a new multi relationship recommendation algorithm, and analyzed the relationship degree between traditional path planning scheme and path planning scheme with three-dimensional spatial information. The experiment shows that the ability of heterogeneous information acquisition of weak relationship recommendation algorithm is better than that of classical recommendation algorithm [6]. Yuan Ling combined with sparse matrix and other related theories to improve particle swarm optimization algorithm, constructed particle swarm optimization algorithm based on image features, and explained the theoretical basis and practical significance of these changes in algorithm performance with game theory. Experiments show that the improved algorithm has better image information extraction performance [7]. Jing Z proposed a hierarchical image processing scheme design and information integration method. Through the research and analysis of different types of image information, it is found that the algo-

*Corresponding author: Yuguang Ye, Quanzhou Normal University, Quanzhou, Fujian, 362000, China,

E-mail: chinaye153@163.com 
rithm can improve the overall convenience of real information of traffic image [8]. Wang et al. Proposed a new vehicle detection image information analysis system based on hyperchaotic mapping, and verified the effectiveness of the system through practice, which is suitable for solving the problem of traffic congestion [9]. Yadollah rasekhipour uses the transformed chaotic sequence to scramble the position of the original road image processing method. This innovative training system has good overall coordination effect [10]. Xianju Fei proposed a new hyperchaotic neural network image processing algorithm to generate real-time spatial position data stream in vehicle navigation. The results show that compared with other related algorithms, the algorithm has the advantages of strong stability and anti-interference, and is suitable for vehicle navigation in complex road conditions [11]. Xinyu Zhang proposed a panoramic vehicle image analysis algorithm. The results show that the algorithm can effectively reduce the road condition error of the driver in the vehicle driving, improve the accuracy of the navigation system, and improve the driver's travel experience [12]. Hulin Kuang proposed an image processing algorithm based on Fourier function, and the results show that the algorithm can effectively reduce the occurrence rate of excessive position deviation events in the process of vehicle navigation [13]. Sun x proposed an automatic generation method of intelligent road navigation scheme based on genetic algorithm and particle swarm optimization algorithm. Experiments show that the algorithm can effectively improve the practicability of image information processing [14]. By simulating the "posture trend diagram" in the vehicle image, Xu Chen realizes the position reference evaluation basis in the vehicle image, and improves the real-time update efficiency of the traditional vehicle image processing scheme [15]. To sum up, it can be seen that most of the current vehicle navigation and path planning modes do not involve the intelligent image processing algorithm based on the image information of reference objects around the road conditions, and there is no research on the real-time image acquisition of road conditions and the construction of relevant models for Intelligent Vehicles [16]. In this context, taking vehicle detection image as the research object, this paper proposes a sparse representation optimization method of image Gaussian mixture based on convolutional neural network, which is mainly divided into three parts. The first part introduces the research status of common vehicle navigation schemes and factors influencing travel experience at home and abroad [17]. In the second part, the sparse representation system of image Gaussian mixture based on convolutional neural network is constructed, and the Gaussian random factor method is used to construct the evaluation index system of image sparse representation [18]. In the third part, the evaluation indexes of the image processing and sparsity representation model are tested and the conclusion is drawn.

\section{Application of convolution neural network in sparse representation of image features}

A typical neural network structure is a feedforward network with three or more layers without feedback and interconnection (the typical three-layer neural network structure is shown in Figure 1). The first and last layers are called input layer and output layer respectively, and the middle layers are hidden layer (also called middle layer).

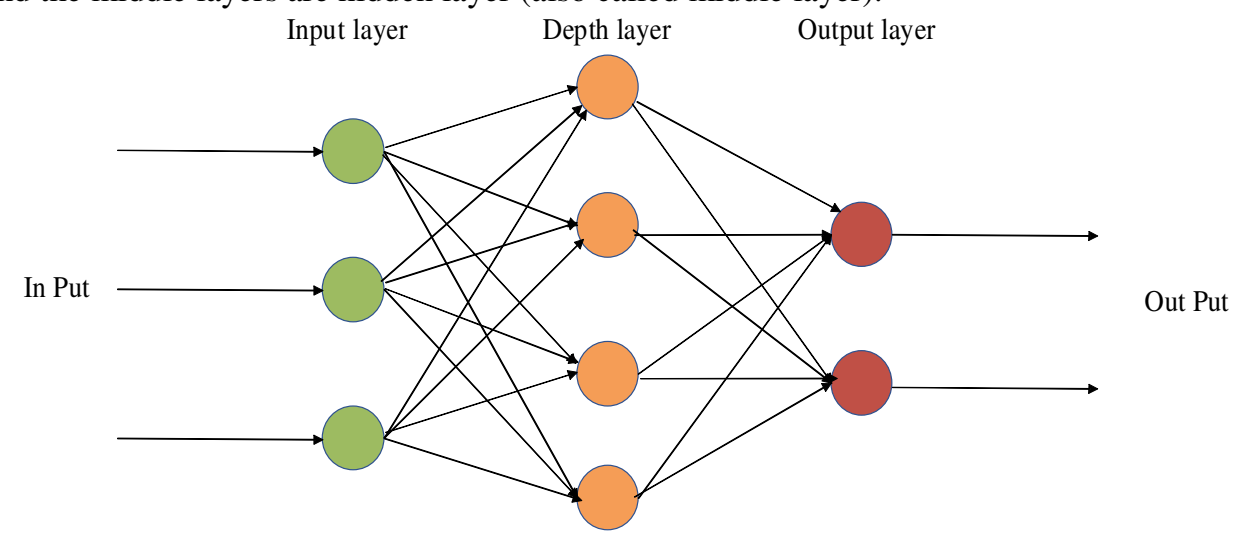

Fig. 1. Three-layer typical structure of neural network

In the neural network, the neurons in each layer are fully connected, while the neurons in each layer are not connected. The neural network algorithm used in the sparse representation of image is an intelligent algorithm with "human biological characteristics" based on the overall structure of human neurons and the brain's direct two-way regulation and automatic processing of neurons. When we carry out random processing, mutual coupling analysis of bidirectional image information classification and vector processing analysis of multiple coupling combinations for a single neuron structure with multiple neuron structure (synapse) characteristics, we can achieve the optimization of multiple software types and reusable framework processing of software development [19]. If the image type with high modularity requirement is found from the total objects to be processed, the probability of being selected for secondary or multiple analysis is high. On the contrary, the probability of the object with low image feature significance being selected for secondary or multiple reusable frame processing is low. The new generation of targets with the characteristics of unified image processing generated by multiple comparative analysis not only inherits the local information of the last round of intelligent filtering, but also is better than the previous generation [20]. In this way, after several two-way information interaction cycles, the sparse representation individuals of image mixed features in line with the reusable framework are finally generated, that is, modular unified processing, local optimization and feature extraction are carried out for a certain type of image. 


\section{Sparse representation model of multi-dimensional image Gaussian mixture features based on convolutional neural network}

\subsection{Basic idea of sparse representation of image Gaussian mixture features based on convolutional neural network}

With the development of artificial intelligence technology, in the aspect of image data processing, the analysis algorithm based on convolution neural network has become a research hotspot and development trend in the fields of information mining and analysis of data association degree, Generally speaking, it is a process in which data are classified into one group according to the similarity or contribution degree of the target task, and the relevant data information or the difference of contribution degree are classified into different groups. "Convolutional neural network is also a basic method of self-learning and updating at the data level. In this paper, the observation object is multi data, and the observation index is the management degree between and within multi data [21-23]. The sparse representation process of different data types is shown in Figure 2.

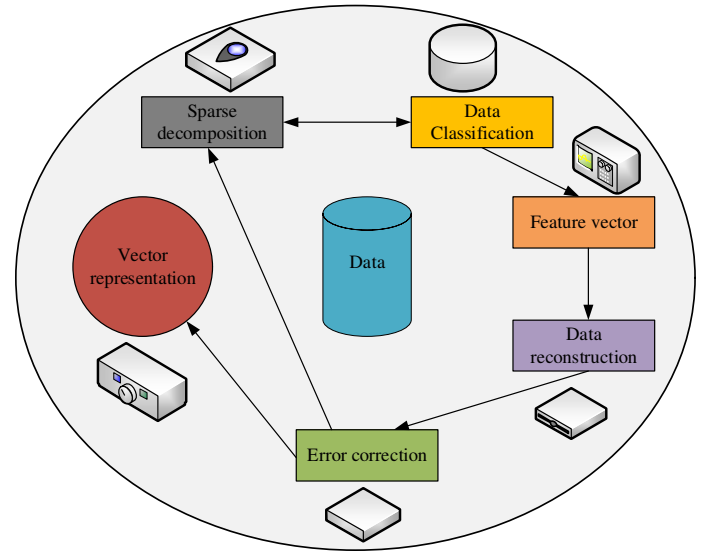

Fig. 2. Sparse representation process for different data types

\subsection{Establishment process of multiple grey convolution neural network based on data mining}

By checking many factors of multiple data, we can judge whether there are closely related factors in multiple data. In this way, we can not only use the comprehensive average index of these closely related factors or one of them to represent multiple such factors, It can also make the information carried by the multi data that need to be processed not be seriously distorted. Firstly, we assume that there are $n$ data objects (local information of image) to be processed, which are called implementation objects, and each observation object has $m$ feature data (feature information of image), the sequence can be obtained as follows:

$$
\begin{aligned}
& X_{1}=\left(x_{1}(1), x_{1}(2), \mathrm{L}, x_{1}(n)\right) \\
& X_{2}=\left(x_{2}(1), x_{2}(2), \mathrm{L}, x_{2}(n)\right)
\end{aligned}
$$

$$
X_{m}=\left(x_{m}(1), x_{m}(2), \mathrm{L}, x_{m}(n)\right)
$$

Then, for all $i \leq j, i, j=1,2, \mathrm{~L}, m$, the absolute correlation degree $\varepsilon_{i j}$ between $X_{i}$ and $X_{j}$ can be calculated, and the calculation formula is:

The upper triangular matrix is obtained:

$$
\varepsilon=\left|\frac{X_{m}+X_{m+1}}{X_{m}-X_{m-1}}\right|
$$

$$
F=\left[\begin{array}{cccc}
\varepsilon_{11} & \varepsilon_{12} & \mathrm{~L} & \varepsilon_{1 m} \\
& \varepsilon_{22} & \mathrm{~L} & \varepsilon_{2 m} \\
& & \mathrm{O} & \mathrm{M} \\
& & & \varepsilon_{m m}
\end{array}\right]
$$

Among them, $\varepsilon_{i i}=1 ; i=1,2, \mathrm{~L}, m$. We call $F$ matrix the incidence matrix of characteristic variables. Take the critical value $r \in[0,1]$, the general requirement is $r>0.5$. When $\varepsilon_{i j} \geq r(i \neq j), X_{i}$ and $X_{j}$ are regarded as the same characteristics. In this process, the following results can be obtained from the simulation analysis of data types of different dimensions. The analysis process is shown in Figure 3. 


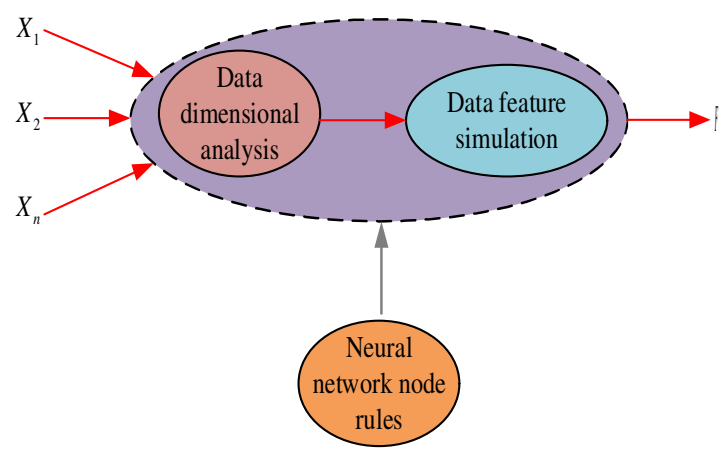

Fig. 3. Simulation analysis process for data types in different dimensions

Next, different data are correlated. We divide the values of analysis indexes of $n$ data objects into $s$ grey groups, which we call $j$ index subclass. The convolution network function of $k$ subclass of $j$ index is denoted as $f_{j}^{k}(\bullet)$. For the convolution neural network image, the discriminant function $H_{j}^{k}(x)$ in the sparse representation analysis model of Gaussian mixture feature is as follows:

$$
H_{j}^{k}(x)=\frac{x+x_{j}^{k}(1)}{x_{j}^{k}(2)-x_{j}^{k}(1)} x \in\left[x_{j}^{k}(1), x_{j}^{k}(2)\right]
$$

Where $x_{j}^{k}(1)$ and $x_{j}^{k}(2)$ are network nodes, $x$ is data type, so the simulation process of sparse representation of Gaussian mixture features of different image types is shown in Figure 4.

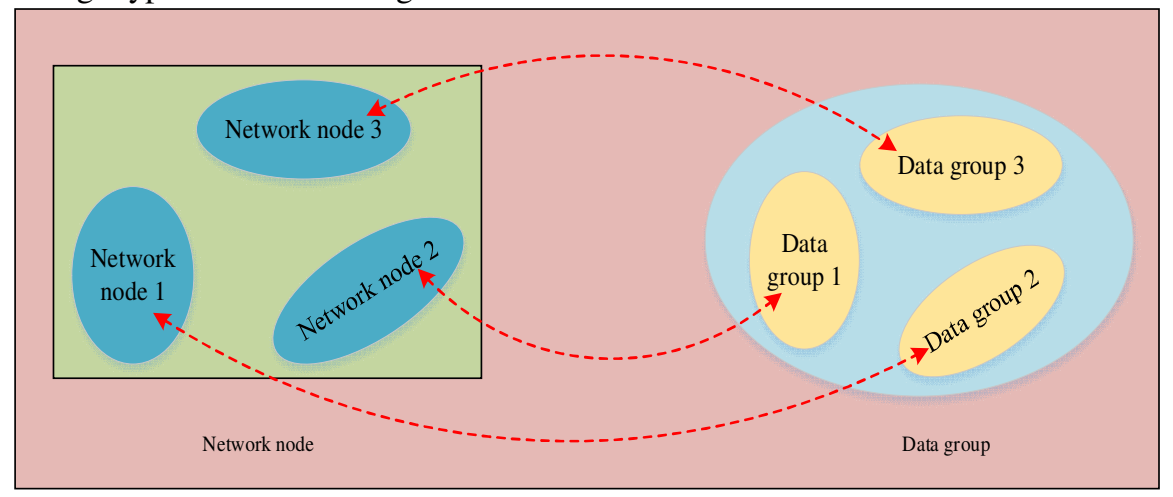

Fig. 4. Gaussian mixture feature sparse representation process of different image types

Correspondingly, if the function $H_{j}^{k}(x)$ has no first and second turning points $x_{j}^{k}(1), x_{j}^{k}(2)$, then $H_{j}^{k}(x)$ is called the convolution network function of lower bound measure, denoted as $H_{j}^{k}\left[-,-, x_{j}^{k}(3), x_{j}^{k}(4)\right]$. In this system, the corresponding lower bound measure function $f_{j}^{k}(x)$ is:

$$
f_{j}^{k}(x)=\frac{x_{j}^{k}(4)-x}{x_{j}^{k}(4)-x_{j}^{k}(3)}, x \in\left[x_{j}^{k}(3), x_{j}^{k}(4)\right]
$$

In addition, if the second and third turning points of the lower limit measure function $f_{j}^{k}(\bullet)$ do not coincide, $f_{j}^{k}(\bullet)$ is called the moderate measure function. The process of simulation analysis using the moderate measure function is shown in Figure 5.

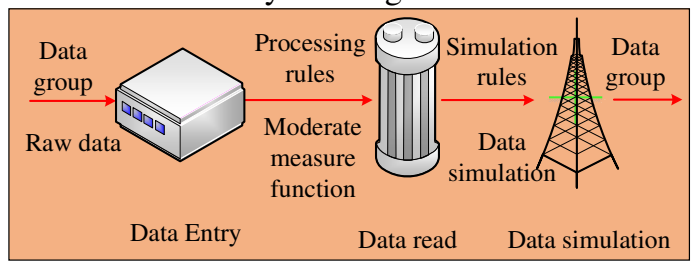

Fig. 5. The result of simulation analysis using moderate measure function

The moderate measure function corresponding to this model is shown in formula 9 . If $f_{j}^{k}(\bullet)$ has no third and fourth turning points, $f_{j}^{k}(\bullet)$ is called the upper limit measure function, denoted as $f_{j}^{k}\left[x_{j}^{k}(1), x_{j}^{k}(2),-,-\right]$. The expression of the whitening weight function of the upper limit measure is shown in formula 10. The output trend of the typical approximate images of the lower limit measure function, the moderate measure function and the upper limit measure function in this model is shown in Figure 6.

$$
Q_{j}^{k}(x)=f_{j}^{k}(x)=\frac{x_{j}^{k}(4)-x}{x_{j}^{k}(4)-x_{j}^{k}(2)}, x \in\left[x_{j}^{k}(2), x_{j}^{k}(4)\right]
$$




$$
W_{j}^{k}(x)=f_{j}^{k}(x)=\frac{x-x_{j}^{k}(1)}{x_{j}^{k}(2)-x_{j}^{k}(1)}, x \in\left[x_{j}^{k}(1), x_{j}^{k}(2)\right]
$$

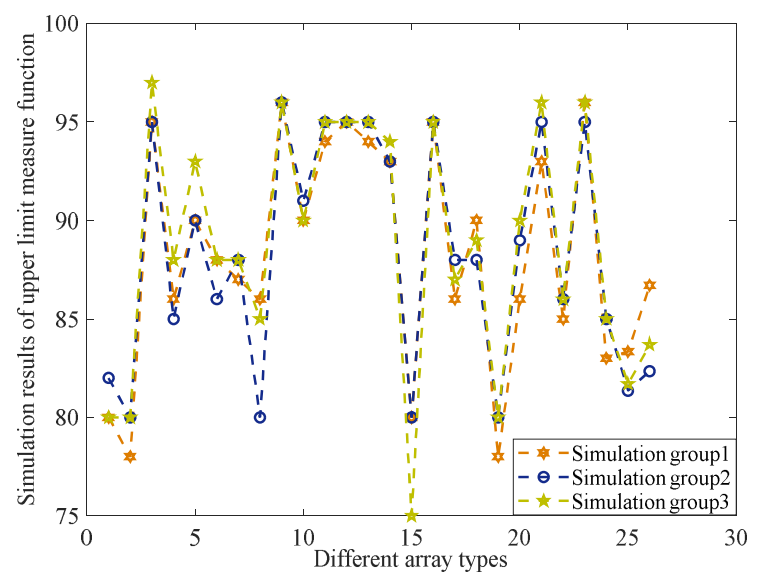

Fig. 6. The output trend of the approximate image in this model

\subsection{Data processing of sparse representation optimization model of Gaussian mixture feature based on convolution neural network}

In this model, in order to better determine the specific meaning of the judgment index corresponding to the image data in the process of gray linear analysis, by determining the convolution variable weight linear coefficient, we can better improve the accuracy of image data mining. Therefore, the weighted linear coefficient $\sigma_{i}^{k}$ can be expressed as:

$$
\sigma_{i}^{k}=\sum_{j=1}^{m} f_{j}^{k}\left(x_{i j}\right) \bullet \eta_{j}^{k}
$$

Specifically, there are:

$$
\sigma_{i}=\left(\sigma_{i}^{1}, \sigma_{i}^{2}, \mathrm{~L}, \sigma_{i}^{s}\right)=\left(\sum_{j=1}^{m} f_{j}^{1}\left(x_{i j}\right) \bullet \eta_{j}^{1}, \sum_{j=1}^{m} f_{j}^{2}\left(x_{i j}\right) \bullet \eta_{j}^{2}, \mathrm{~L}, \sum_{j=1}^{m} f_{j}^{s}\left(x_{i j}\right) \bullet \eta_{j}^{s}\right)
$$

The above formula is called linear coefficient of multilayer convolutional neural network, and its corresponding linear coefficient matrix is:

$$
\Sigma=\left(\sigma_{i}^{k}\right)=\left[\begin{array}{llll}
\sigma_{1}^{1} & \sigma_{1}^{2} & \mathrm{~L} & \sigma_{1}^{s} \\
\sigma_{2}^{1} & \sigma_{2}^{2} & \mathrm{~L} & \sigma_{2}^{s} \\
\mathrm{~L} & \mathrm{~L} & \mathrm{~L} & \mathrm{~L} \\
\sigma_{n}^{1} & \sigma_{n}^{2} & \mathrm{~L} & \sigma_{n}^{s}
\end{array}\right]
$$

In order to realize the data mining of convolutional neural network linear analysis process, it is necessary to translate the information of target data into language information that can be recognized by computer through certain patterns [24]. Then, through the computer database information and the preset multi data multi gray linear judgment program, some data information is deeply mined and analyzed, so as to realize the quadratic linear processing of the data after the first linear analysis [25-27]. Therefore, the simulation results of Gaussian mixture feature sparse representation of image data are shown in Figure 7.

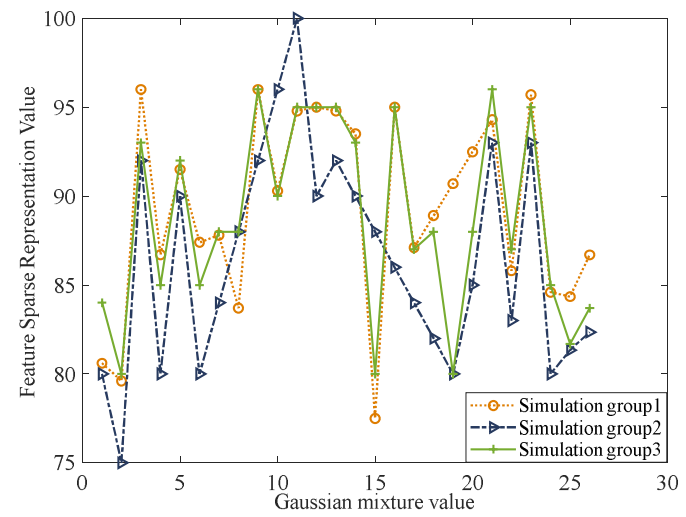

Fig. 7. Simulation process of image feature sparse representation

Finally, some low-frequency or meaningless image data information is deleted or removed purposefully, and the process is stored through a specific mode, so as to facilitate the data restoration required by the later error handling operation, thus forming a 
special data information record, which realizes the conversion of data information into computer storage information, Its storage function can be expressed as $T(x)$.

$$
T(x)=\frac{\sum_{j=1}^{m} f_{j}^{1}\left(x_{j}\right)}{\eta_{j}^{1}}
$$

Where, $\eta_{j}^{1}$ is the storage coefficient, and its sparse representation can be expressed as $T^{\prime}(x)$, which can be expressed as:

$$
T^{\prime}(x)=\frac{\sum_{j=1}^{m} f_{j}^{1}\left(x_{j}\right)}{\eta_{j}^{1} H(x)}
$$

Where $\eta_{j}^{1}$ is the storage coefficient and $H(x)$ is the discriminant function.

Choosing different gray linear methods will make the data in the cluster have different correlation degree. Convolution neural network analysis model is to search and filter unknown data objects (newly acquired image information) through data mining of multi-target data [28-30]. The screening function is $P(x)$, and the expression is:

$$
P(x)=\frac{\sum_{j=1}^{m}\left(x_{j}-\bar{x}\right)}{\eta_{j}^{1} \bar{x}}
$$

Where, $\eta_{j}^{1}$ is the storage coefficient.

When it is necessary to classify different types of data indicators (such as the related assessment data indicators of a certain geographical location), the convolution neural network evaluation model based on trigonometric function is adopted, and its evaluation model $Z(x)$ can be expressed as:

$$
Z(x)=\frac{1}{\eta_{j}^{1} \sum_{j=1}^{m}\left(x_{j}-\bar{x}\right)}
$$

Where $\eta_{j}^{1}$ is the storage coefficient and $\bar{x}$ is the average number of image decomposition information.

$$
Z(x)=\frac{1}{\eta_{j}^{1} \sum_{j=1}^{m}\left(x_{j}-\bar{x}\right)}
$$

In the optimal case, convolution neural network analysis model can achieve a certain degree of similarity between a variety of data recognition. However, there are still some problems in the process of data mining and image processing algorithm. In order to improve the recognition degree of image data information (feature information) and a variety of data (image color channel, salient features, etc.) by multiple gray linear analysis model, the expression of association degree $R(x)$ is:

$$
R(x)=\frac{\eta_{j}^{1}}{\sum_{j=1}^{m}\left(\frac{x_{j}-\bar{x}}{x_{j+1}+\bar{x}}\right)}
$$

At present, the most commonly used method is to achieve accurate linear analysis through big data database statistics and data comparison between the same image types. The well-known information comparison and judgment of image feature recognition information and sparse representation pattern also use the above method, and the comparison simulation results are shown in Figure 8 .

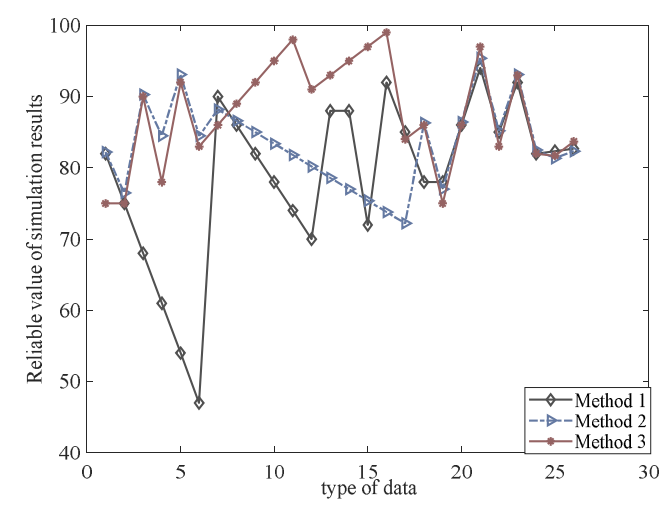

Fig. 8. Comparison of simulation results of different methods 


\section{Result analysis and discussion}

\subsection{Experimental design process and data results}

When we evaluate the signal sparsity of vehicle detection image, we need to evaluate it from many aspects. According to the driver's needs, we propose 15 indicators to evaluate the quality of sparse representation of Gaussian mixture features. Through the observation results of 15 relevant data of image Gaussian mixture features, the above indicators are properly classified and sparsely characterized. By deleting some unnecessary (i.e. less influential) indicators, the characterization standard becomes simplified, so as to achieve the quantitative representation of the above data indicators, which is more convincing [31]. Through the analysis of 9 different vehicle detection images, the experimental results are shown in Table 1.

Table 1

Scores for each indicator corresponding to 9 samples

\begin{tabular}{c|c|c|c|c|c|c|c|c|c|c|c}
\hline & 1 & 2 & 3 & 4 & 5 & 6 & 7 & 8 & 9 \\
\hline $\mathrm{X} 1$ & 6 & 9 & 7 & 5 & 6 & 7 & 9 & 9 \\
\hline $\mathrm{X} 2$ & 2 & 5 & 3 & 8 & 8 & 7 & 8 & 9 & 7 \\
\hline $\mathrm{X} 3$ & 5 & 8 & 6 & 5 & 8 & 6 & 8 & 8 & 8 \\
\hline $\mathrm{X} 4$ & 8 & 10 & 9 & 6 & 4 & 8 & 8 & 9 & 8 \\
\hline $\mathrm{X} 5$ & 7 & 9 & 8 & 5 & 4 & 7 & 8 & 9 & 8 \\
\hline $\mathrm{X} 6$ & 8 & 9 & 9 & 9 & 9 & 10 & 8 & 8 & 8 \\
\hline $\mathrm{X} 7$ & 8 & 10 & 7 & 2 & 2 & 5 & 8 & 8 & 5 \\
\hline $\mathrm{X} 8$ & 3 & 5 & 4 & 8 & 8 & 9 & 10 & 10 & 9 \\
\hline $\mathrm{X} 10$ & 9 & 9 & 9 & 4 & 5 & 6 & 8 & 9 & 8 \\
\hline $\mathrm{X} 11$ & 7 & 10 & 8 & 6 & 8 & 7 & 9 & 9 & 9 \\
\hline $\mathrm{X} 12$ & 7 & 8 & 8 & 8 & 8 & 8 & 8 & 9 & 9 \\
\hline $\mathrm{X} 13$ & 5 & 8 & 6 & 7 & 8 & 6 & 9 & 9 \\
\hline $\mathrm{X} 14$ & 7 & 8 & 8 & 6 & 7 & 6 & 8 & 9 & 9 \\
\hline
\end{tabular}

The experimental results are obtained by solving the relevant data values of the characteristic variables of the above 15 data indexes as shown in Figure 9.

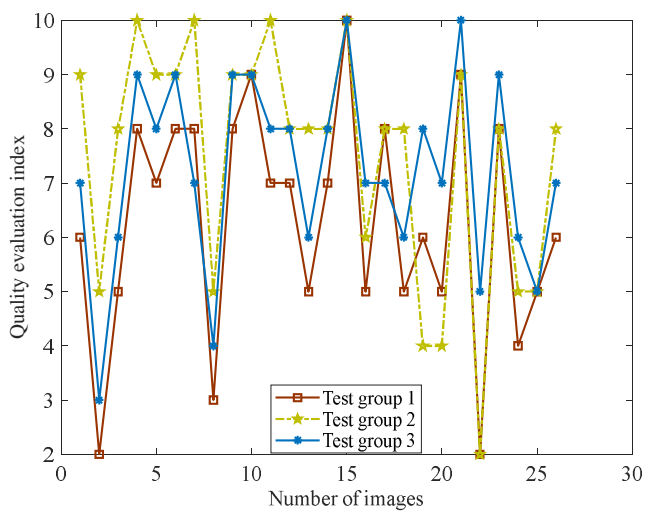

Fig. 9. Preliminary experimental results and indicators

According to the incidence matrix in formula 4, the data index incidence matrix as shown in Table 2 is obtained.

Table 2

Relevance of 15 data out of 9 samples after correlation matrix operation

\begin{tabular}{|c|c|c|c|c|c|c|c|c|c|c|c|c|c|c|c|}
\hline & $\mathrm{X} 1$ & $\mathrm{X} 2$ & X3 & $\mathrm{X} 4$ & X5 & X6 & X7 & X8 & X9 & X10 & X11 & X12 & X13 & X14 & X15 \\
\hline $\mathrm{X} 1$ & 1 & .66 & .88 & .52 & .58 & .77 & .51 & .66 & .51 & .51 & .9 & .88 & .8 & .67 & .51 \\
\hline X2 & & 1 & .07 & .51 & .53 & .59 & .5 & .99 & .51 & .51 & .63 & .62 & .77 & .55 & .51 \\
\hline X3 & & & 1 & .56 & .7 & .51 & .72 & .51 & .51 & .51 & .8 & .78 & .9 & .63 & .51 \\
\hline $\mathrm{X} 4$ & & & & 1 & .56 & .53 & .58 & .51 & .69 & .62 & .52 & .52 & .51 & .54 & .6 \\
\hline X5 & & & & & 1 & .07 & .51 & .53 & .53 & .52 & .61 & .61 & .55 & .75 & .52 \\
\hline X6 & & & & & & 1 & .51 & .59 & .05 & .52 & .84 & .86 & .66 & .81 & .51 \\
\hline X7 & & & & & & & 1 & .5 & .7 & .83 & .51 & .51 & .51 & .51 & .89 \\
\hline X8 & & & & & & & & 1 & .51 & .51 & .63 & .62 & .77 & .55 & .51 \\
\hline X9 & & & & & & & & & 1 & .81 & .52 & .52 & .51 & .53 & .76 \\
\hline X10 & & & & & & & & & & 1 & .51 & .51 & .51 & .52 & .92 \\
\hline X11 & & & & & & & & & & & 1 & .97 & .74 & .71 & .51 \\
\hline
\end{tabular}




\begin{tabular}{c|l|l|l|l|l|l|l|l|l|l|l|l|l|l|l}
\hline $\mathrm{X} 12$ & & & & & & & & & & & & 1 & .73 & .72 & .51 \\
\hline $\mathrm{X} 13$ & & & & & & & & & & & & & 1 & .6 & .51 \\
\hline $\mathrm{X} 14$ & & & & & & & & & & & & & & 1 & .52 \\
\hline $\mathrm{X} 15$ & & & & & & & & & & & & & & & 1 \\
\hline
\end{tabular}

\subsection{Experimental results analysis of sparse representation of image Gaussian mixture features based on convolutional neural network}

From table 1 and table 2, we can know that some or some of the above 15 data indicators for evaluating the quality of the algorithm do have correlation or confusion. From the data matrix in Table 3, we can know that the correlation degree between the first index and the 11th index is 0.9 , and through the nine related test indexes of the navigation scheme, it further proves that the multi data multi analysis model can achieve the deep mining of data in the process of processing the related image processing data. The correlation degree between the 10 th index and the 15 th index is 0.92 , which is very close to 1 . It also shows that there is a strong correlation between the two indexes, which can explain the internal relationship between image Gaussian mixture feature and image sparse representation optimization. In addition, we can see from table 3 that the correlation between the second and third indicators is 0.07 , which is very small and even negligible. This is also in line with our life experience. It once again shows the application accuracy of the convolutional neural network in the sparse representation algorithm of Gaussian mixture features of images.

Table 3

The partial correlation between different data indicators

\begin{tabular}{c|c}
\hline Related indicators & Degree of association \\
\hline The first indicator and the eleventh indicator & 0.90 \\
\hline Item 10 and Item 15 & 0.92 \\
\hline The second and third indicators & 0.07 \\
\hline
\end{tabular}

4.3. Summary of experimental results analysis

The innovation of this paper is to combine image Gaussian mixture feature sparse representation method and neural network algorithm to form an adaptive image sparse representation feature model with adaptive capabilities, which is used for adaptive sparse hybrid representation of different image data centers. On this basis, it can make full use of the information source of each image data in the database and the characteristic information required by different image types to achieve the overall approach and unified management of the image data information database, and then use the neural network correlation factor to quantify Describe the similarity between the comparison columns (different types of image data flow demand data) and the reference column (the Gaussian mixture data column through preliminary screening) and the degree of agreement between the expected index (the upper limit of the image sparse representation method), and the quantified index is used to complete the comparison. The formulation of image types can efficiently realize the formulation of optimization schemes for different image types through different sparse representation methods.

In the process of constructing a convolutional neural network-based image sparse representation and learning analysis model, this research adopts a neural network optimization algorithm based on local search for optimal solutions, combined with a machine learning model, and selects 4 sparse representations that are mixed with the image to optimize efficiency Related characteristic parameters.

It can be seen from the experimental results that in the process of processing the experimental data, a highly efficient and intelligent data analysis method is adopted. This model can realize the sparse representation data of different images and the data of different types of image representation methods. For unified management, according to the actual needs of local differences and sparse representation of data, algorithms are used for intelligent optimization and analysis processing to achieve high-precision utilization of data and remote dynamic maintenance. After that, the eigenvalues of the signals collected by the wireless nano data processing equipment and the structural characteristics of different images in the sparse representation process are used according to the different feature description values such as the vector difference and matrix difference shown by the machine. The intelligent optimization processing and in-depth information mining process of learning model and neural network algorithm realizes the classification of different image Gaussian mixture feature sparse representation methods in the data analysis link.

Finally, through the neural network algorithm in the process of image sparse representation, a series of data analysis processes are classified according to the difference of information, so as to achieve a high degree of similarity of different data, and according to the sparse representation of different images With different requirements, it can realize the fitting analysis and simulation of similar or identical data, and then complete the high-efficiency and high-precision classification of the sparse representation optimization link of the image Gaussian mixture feature.

\section{Conclusion}

In the aspect of sparse representation optimization of Gaussian mixture features of image, due to the large amount of data and heavy burden of data analysis, there are some problems in the aspect of Gaussian mixture features representation of image, such as 
insufficient accuracy and high error rate of navigation scheme. Based on this, this paper proposes a sparse representation system of image Gaussian mixture features based on convolutional neural network. Firstly, a sparse representation of image Gaussian mixture features based on convolution neural network is proposed for real-time sensing of road condition feature parameters. Secondly, the hierarchical framework and index relationship of sparse representation of image features are clearly defined. Finally, 15 indexes are selected to experiment on 9 different images. The experimental results show that this algorithm can realize the recognition and mining of the internal information and external correlation degree between different data, and can effectively solve the problems of low accuracy, sparse features and poor representation in traditional image processing. However, this algorithm only analyzes the influence and correlation degree from the aspect of image local analysis, and does not take other potential factors of image feature sparse representation into consideration. Therefore, the comprehensive analysis of this index evaluation system and the influence degree of other factors need to be further studied.

\section{Declarations \\ Funding}

No funding is received.

Research involving Human Participants and/or Animals:

Not applicable

Informed consent:

Not applicable

Conflicts of interests:

The authors declare that they have no conflicts of interest.

Availability of data and material:

No data available.

Code availability

Not Applicable.

Authors' Contributions

Yuguang Ye is responsible for planning, applying the methodology, deduced the results, writing and editing the manuscript.

\section{References}

[1] Yufeng, Li, Wei, He. Research on SAR image change detection algorithm based on hybrid genetic FCM and image registration. Multimedia Tools and Applications, 76(13) (2018), 15137-15153.

[2] Han Gaining, Fu Weiping, Wang Wen. The Lateral Tracking Control for the Intelligent Vehicle Based on Adaptive PID Neural Network. Sensors, 17(6) (2018), 1244-1249.

[3] Chen, JiaWang, Zhu, Huangchao, Zhang, Lei. Research on fuzzy control of path tracking for underwater vehicle based on genetic algorithm optimization. Ocean Engineering, 15(6) (2018), 217-223.

[4] Xiang T, Wang H. Research on Distributed 5G Signal Coverage Detection Algorithm Based on PSO-BP-Kriging. Sensors (Basel, Switzerland), 18(12) (2018).

[5] Pan, Hailan, Lei, Yongmei, Jian, Chen. Research on digital image encryption algorithm based on double logistic chaotic map. EURASIP Journal on Image and Video Processing, (2018), (1).

[6] Miao Q, Cebon D. Path-Following Control Based on Ground-Watching Navigation. IEEE Transactions on Intelligent Transportation Systems, (99) (2017), 1-11.

[7] Yuan Ling, Li Dan, Wei Shikang. Research of Deceptive Review Detection Based on Target Product Identification and Metapath Feature Weight Calculation. Complexity, (2018), 1-12.

[8] Jing Z, Yu H, Hu M. Research on A PM Slotless Linear Generator Based on Magnet Field Analysis Model for Wave Energy Conversion. IEEE Transactions on Magnetics, (99) (2017), 1-1.

[9] Wang, Shaohua, Yu, Chengquan, Shi, Dehua. Research on Speed Optimization Strategy of Hybrid Electric Vehicle Queue Based on Particle Swarm Optimization. Mathematical Problems in Engineering, (2018), 1-14.

[10] Yadollah Rasekhipour, Amir Khajepour, Shih-Ken Chen. A Potential Field-Based Model Predictive Path-Planning Controller for Autonomous Road Vehicles. IEEE Transactions on Intelligent Transportation Systems, (99) (2016), 1-13.

[11] Xianju Fei, Guozhong Tian. Research on data mining algorithm based on neural network and particle swarm optimization. Journal of Intelligent \& Fuzzy Systems, 35(4) (2018), 1-6.

[12] Xinyu Zhang, Ding Liu, Simi Wang. Shape Detection of Silicon Single Crystal Based on the MUSIC Algorithm. IEEE Sensors Journal, (99) (2019), 1-1.

[13] Hulin Kuang, Xianshi Zhang, Yong-Jie Li. Nighttime Vehicle Detection Based on Bio-Inspired Image Enhancement and Weighted Score-Level Feature Fusion. IEEE Transactions on Intelligent Transportation Systems, 18(99) (2016), 1-10.

[14] Sun X, Zhang H, Cai Y. Hybrid modeling and predictive control of intelligent vehicle longitudinal velocity considering nonlinear tire dynamics. Nonlinear Dynamics, 97(1) (2019), 1-16.

[15] Xu Chen, Lei Liu, Yubin Deng. Vehicle detection based on visual attention mechanism and adaboost cascade classifier in intelligent transportation systems. Optical and Quantum Electronics, 51(8) (2019).

[16] Luke P, Mcewen J D, Mayeul D. Robust sparse image reconstruction of radio interferometric observations with PURIFY. Monthly Notices of the Royal Astronomical Society, 473(1) (2018), 1038-1058.

[17] Li Y, Dong W, Member. Image Super-resolution with Parametric Sparse Model Learning. IEEE Transactions on Image Processing, 27(9) (2018), 4638-4650. 
[18] A K L, A S Q, B H Y. Extensible image object co-segmentation with sparse cooperative relations - ScienceDirect. Information Sciences, 521 (2020), 422-434.

[19] Chen G, Wang F, Qu S. Pseudo-Image and Sparse Points: Vehicle Detection With 2D LiDAR Revisited by Deep Learning-Based Methods. IEEE Transactions on Intelligent Transportation Systems, (2020), 1-13.

[20] Sheng L, Yuan F, Zhang S. \$L_0\$ Sparse Regularization-Based Image Blind Deblurring Approach for Solid Waste Image Restoration. IEEE Transactions on Industrial Electronics, 66(12) (2019), 9837-9845.

[21] Ha I Y, Wilms M, Handels H. Model-Based Sparse-to-Dense Image Registration for Realtime Respiratory Motion Estimation in Image-Guided Interventions. IEEE Transactions on Biomedical Engineering, 66(2) (2019), 302-310.

[22] Jian J, Ren F, Ji H F. Generalised non-locally centralised image de-noising using sparse dictionary. Iet Image Processing, 12(7) (2018), 1072-1078.

[23] A F S, D T K A, Jürgen Endres a b. Temporal and volumetric denoising via quantile sparse image prior. Medical Image Analysis, 48 (2018), 131-146.

[24] Wang W, Zhao M, Wang J. Effective android malware detection with a hybrid model based on deep autoencoder and convolutional neural network. Journal of Ambient Intelligence and Humanized Computing, 10(8) (2019), 3035-3043.

[25] You W, Shen C, Guo X. A hybrid technique based on convolutional neural network and support vector regression for intelligent diagnosis of rotating machinery. Advances in Mechanical Engineering, 9(6) (2017), 1687814017704146.

[26] Geng Z, Wang Y. Automated design of a convolutional neural network with multi-scale filters for cost-efficient seismic data classification. Nature communications, 11(1) (2020),1-11.

[27] Zheng Y, Zhen B, Chen A. A hybrid convolutional neural network for super-resolution reconstruction of MR images. Medical physics, 47(7) (2020), 3013-3022.

[28] Özyurt F, Tuncer T, Avci E. A novel liver image classification method using perceptual hash-based convolutional neural network. Arabian Journal for Science and Engineering, 44(4) (2019), 3173-3182.

[29] Kilicarslan S, Adem K, Celik M. Diagnosis and classification of cancer using hybrid model based on ReliefF and convolutional neural network. Medical hypotheses, 137 (2020), 109577.

[30] Ilyas N, Shahzad A, Kim K. Convolutional-neural network-based image crowd counting: review, categorization, analysis, and performance evaluation. Sensors, 20(1) (2020), 43.

[31] El-Sawy A, Loey M, El-Bakry H. Arabic handwritten characters recognition using convolutional neural network. WSEAS Transactions on Computer Research, 5 (2017), 11-19.

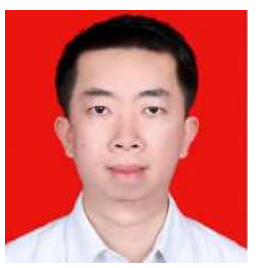

YuGuang Ye, Master of social, Associate Professor. Graduated from Huaqiao University in 2007. Worked in Quanzhou Normal University. His research interests include computer network, Internet of Things systems, and image processing, Email address:chinaye153@163.com 